\title{
A REMARK ON COSINE FAMILIES
}

\author{
SAMUEL M. RANKIN III
}

\begin{abstract}
Let $C(t), t \in R$, be a strongly continuous cosine family and $A$ its infinitesimal generator. Then the set $E \stackrel{\text { def }}{=}\{x \in X: C(t) x$ is once continuously differentiable in $t$ on $R$ \} of the Banach space $X$ is contained in the domain of $(-A)^{\alpha}$ for $0<\alpha<1 / 2$.
\end{abstract}

The purpose of this note is to prove for a strongly continuous cosine family $C(t)$, $t \in R$, defined on a Banach space $X$ and with infinitesimal generator $A$, that the set $E \stackrel{\text { def }}{=}\{x \in X: C(t) x$ is once continuously differentiable in $t$ on $R\}$ is contained in the set $D\left[(-A)^{\alpha}\right], 0 \leqslant \alpha<1 / 2$. The set $D\left[(-A)^{\alpha}\right]$ is the domain of the $\alpha$ power of the operator $-A$.

A one parameter family $C(t), t \in R$, of bounded linear operators mapping the Banach space $X$ into itself is called a strongly continuous cosine family if and only if

$$
\begin{aligned}
& C(t+s)+C(t-s)=2 C(t) C(s) \text { for all } s, t \in R, \\
& C(0)=I, \\
& C(t) x \text { is continuous in } t \in R \text { for each fixed } x \in X .
\end{aligned}
$$

The associated sine family is given by $S(t) x=\int_{0}^{t} C(s) x d s$ for $x \in X$ and $t \in R$. The linear operator $A: X \rightarrow X$ defined by $A x=C^{\prime \prime}(0) \dot{x}$ and with dense domain $D(A)=\{x \in X: C(t) x$ is twice continuously differentiable in $t$ on $R\}$ is called the infinitesimal generator of $C(t), t \in R$. For other properties of cosine families used in this paper see [2] or [9].

The following theorem appears in C. Travis and G. Webb [8] and [9]:

THEOREM 1. Let $C(t), t \in R$, be a strongly continuous cosine family with associated sine family $S(t), t \in R$, and infinitesimal generator $A$. The following statements are equivalent:

(i) there exists a closed linear operator $B$ on $X$ such that $B^{2}=A$ and $B$ commutes with every operator in $B(X, X)$ which commutes with $A ; S(t)$ maps $X$ into $D(B)$ for each $t \in R ; B S(t) x$ is continuous in $t \in R$ for each fixed $x \in X$;

(ii) $E=D(B)$, the domain of $B$.

The conditions stated in part (i) of Theorem 1 have also been considered by $\mathbf{H}$. Fattorini in [2] and [3]. Fattorini has shown in [3] that every strongly continuous cosine family defined on the Banach space $L^{p}, 1<p<\infty$, satisfies condition (i).

Presented to the Society, January 25, 1979; received by the editors March 2, 1978 and, in revised form, March 16, 1979.

AMS (MOS) subject classifications (1970). Primary 47D99, 47D05; Secondary 34G05.

Key words and phrases. Cosine family, semigroup of operators, differential equation. 
However, there are cosine families which do not satisfy condition (i) as shown by $\mathrm{J}$. Kisynski [4] and B. Nagy [6]. It remains open whether or not $E \subset D\left[(-A)^{1 / 2}\right]$ in general.

Proposition. Let $A$ be the infinitesimal generator of the strongly continuous cosine family $C(t), t \in R$, such that there exists an $M>0$ with $\left\|(\lambda-A)^{-1}\right\| \leqslant M / \lambda$ for all $\lambda>0$. Then the set $E$ is contained in $D\left[(-A)^{\alpha}\right]$ for $0 \leqslant \alpha<1 / 2$.

Proof. It is known, V. Balakrishman [1], that $-(-A)^{1 / 2}$ generates an analytic semigroup $T(t)$ with $T(t) X \subset D(A)$. Now if $x \in E$ then $S(t) x$ is twice continuously differentiable and the function

$$
u(t)=\int_{0}^{t} T(t-v) S(v) x d v
$$

solves the differential equation

$$
u^{\prime}(t)=-(-A)^{1 / 2} u(t)+S(t) x, \quad u(0)=0 .
$$

Following a change of variable in the integral, we have

$$
u^{\prime}(t)=\int_{0}^{t} T(v) C(t-v) x d v
$$

and

$$
\begin{aligned}
u^{\prime \prime}(t) & =\int_{0}^{t} T(v) A S(t-v) x d v+T(t) x=\int_{0}^{t} T(t-v) A S(v) x d v+T(t) x \\
& =A u(t)+T(t) x=-(-A)^{1 / 2} \int_{0}^{t} T(t-v) C(v) x d v+C(t) x \\
& =-(-A)^{1 / 2} u^{\prime}(t)+C(t) x, \quad t>0 .
\end{aligned}
$$

We can now write for $t>0$

$$
\begin{aligned}
A u(t)+T(t) x & =-(-A)^{1 / 2}\left[-(-A)^{1 / 2} u(t)+S(t) x\right]+C(t) x \\
& =-A u(t)-(-A)^{1 / 2} S(t) x+C(t) x,
\end{aligned}
$$

which implies that

$$
C(t) x=2 A u(t)+T(t) x+(-A)^{1 / 2} S(t) x
$$

for all $x \in E$ and $t>0$.

Under the stated conditions the fractional power of the operator $(-A)^{1 / 2}$ exist and $\left\|\left[(-A)^{1 / 2}\right]^{\beta} T(t)\right\| \leqslant C / t^{\beta}$ for all $t>0$, for some $C>0$, and for $0<\beta<1$. (See the book by S. Krein [5] or the lecture notes by A. Pazy [7].)

Therefore since $\int_{0}^{t}\left[(-A)^{1 / 2}\right]^{\beta} T(t-v) A S(v) x d v(t>0)$ exists for any $0 \leqslant \beta<$ 1, we have that $A u(t)=\int_{0}^{t} T(t-v) A S(v) x d v$ is in $D\left[(-A)^{1 / 2}\right]^{\beta}, t>0$, for all $0 \leqslant \beta<1$. This is equivalent to $A u(t) \in D\left[(-A)^{\alpha}\right], t<0$, for all $0 \leqslant \alpha<1 / 2$.

Appealing now to equation (*), the fact that $(-A)^{1 / 2} S(t) x \in D\left[(-A)^{1 / 2}\right], T(t) x \in$ $D(A)$, and $A u(t) \in D\left[(-A)^{\alpha}\right](0 \leqslant \alpha<1 / 2)$, for all $t>0$, we have that $C(t) x \in$ $D\left[(-A)^{\alpha}\right]$ for $t>0$. The identity $x=2 C(t) C(t) x-C(2 t) x$ gives the result, since $C(t), t \in R$, leaves $D\left[(-A)^{\alpha}\right]$ invariant. 
REMARK. The condition $\left\|(\lambda-A)^{-1}\right\|<M / \lambda$ for $\lambda>0$ is not as restrictive as it may seem since by Theorem 2.7 of [9], $\left\|\left(\lambda^{2}-A\right)^{-1}\right\|<M / \lambda(\lambda-w)(\lambda>w)$. If $A$ generates a strongly continuous cosine family then so does $A_{b}=A-b^{2} I$ [2]. Thus if one chooses $b>w$, we have for all $\lambda>0$

$$
\left\|\left(\lambda^{2}-A_{b}\right)^{-1}\right\|=\left\|\left(\lambda^{2}+b^{2}-A\right)^{-1}\right\|<\frac{M}{\lambda\left(\lambda^{2}+b^{2}-w\right)}<\frac{M^{\prime}}{\lambda^{2}} .
$$

Substituting $\sqrt{\lambda}$ for $\lambda$ the condition of the proposition is satisfied by $A_{b}$. In this sense (after a suitable translation) every strongly continuous cosine family satisfies the above proposition.

Acknowledgement. The author would like to thank Professor Glenn Webb for several helpful suggestions regarding the proof of the proposition.

\section{REFERENCES}

1. A. V. Balakrishnan, Fractional powers of closed operators and the semigroups generated by them, Pacific J. Math. 10 (1968), 419-437.

2. H. O. Fattorini, Ordinary differential equations in linear topological spaces. I, J. Differential Equations 5 (1968), 72-105.

3. __ Ordinary differential equations in linear topological spaces. II, J. Differential Equations 6 (1969), 50-70.

4. J. Kisyński, On operator-valued solutions of d'Alemberts' functional equation. II, Studia Math. 42 (1971), 43-66.

5. S. G. Krein, Linear differential equations in Banach space, Transl. Math. Monograph, vol. 29, Amer. Math. Soc., Providence, R.I., 1971.

6. B. Nagy, Cosine operator functions and the abstract Cauchy problem, Period. Math. Hungar. 7 (1976), 15-18.

7. A. Pazy, Semi-groups of linear operators and application to partial differential equations, Lecture Notes, University of Maryland, 1974.

8. C. Travis and G. Webb, Cosine families and abstract nonlinear second order differential equation, Acta Math. Acad. Sci. Hungar 32 (1978), 75-96.

9. Second order differential equations in Banach space, Nonlinear Equations in Abstract spaces, edited by V. Lakshmikantham, 1978, pp. 331-361.

Department of Mathematics, West Virginia University, Morgantown, West Virginia 26506 